\title{
Symmetric photonic molecules formed from coupled microspheres
}

\author{
John F. Donegan*a, Matthias Gerlach ${ }^{\mathrm{b}}$, Yury P. Rakovich ${ }^{\mathrm{b}}$ \\ ${ }^{a}$ Semiconductor Photonics Group, School of Physics and Principal Investigator CRANN Research \\ Centre, Trinity College Dublin, Dublin 2, Ireland; \\ ${ }^{\mathrm{b}}$ Semiconductor Photonics Group, School of Physics, Trinity College Dublin, Dublin 2, Ireland
}

\begin{abstract}
Coherent coupling of optical resonances in spherical microcavities results in the formation of so called photonic molecules. The name originates from the analogy to chemical molecules. The coupled optical modes are similar to the atomic orbitals of a chemical molecule. In this work, we studied the resonance spectrum of the symmetric photonic molecules. Furthermore, symmetric directional emission of light from the photonic molecules is experimentally shown. The photonic molecules are illuminated in the vertical direction with a defocused laser beam. The emission is attributed to photonic nanojets generated in the structure. Spectral analysis exhibit whispering gallery mode resonances of coupled and uncoupled modes. A benzene molecule-like structure consisting of a 7-microspheres cyclic photonic molecule shows a field emission pattern similar to the spatial distribution of the orbitals of the benzene molecule.
\end{abstract}

Keywords: Coupled resonators, micro-optics, photonic molecules, nano-jets, whispering gallery modes

\section{INTRODUCTION}

Dielectric transparent microspheres are three-dimensional spherical microcavities which provide high Q-factors and a small mode volume leading to strong optical feedback within the cavity [1]. The optical resonances, also called whispering gallery modes (WGMs) are caused by total internal reflection of the light at the surface inside the sphere. As the electromagnetic field is not fully confined within the sphere, an evanescent field is present surrounding the spherical particle, which enables unique possibilities for interaction of the optical resonances with the surrounding medium or objects in close proximity to the cavity. Two or more microspheres close to each other allows optical coupling of the modes between the spheres which results in a complex rearrangement of the mode structure in the strong coupling regime similar to the electronic molecular orbitals in a chemical molecule. This leads to the notation of photonic molecules (PMs) [2] for coherently coupled microspheres. The optical modes in a coupled system of microcavities experience splitting into bonding and anti-bonding modes. The so called supermodes in a photonic molecule are WGMs which extend over the whole structure of the coupled microcavities. Photonic molecule structures consisting of three or more microcavities were studied theoretically by different groups with regards to supermodes with dramatically increased Q-factors, low laser threshold and directional emission [3-6]. For optimal results, the structure requires a symmetric photonic molecule with size-matched microcavities and optimised gap alignment between the spheres [6]. Apart from microcavities arranged in arrays, chains of coupled microspheres have also been studied for waveguiding purposes [7, 8]. In our recent studies of photonic molecules made of microspheres, we investigated optical coupling of a bi-sphere system. We observed the fine structure of the coherently coupled non-degenerate modes [9]. The optical coupling results in a splitting of the azimuthal resonances. In this present work, we studied the optical properties of symmetrically arranged microspheres with regards to coupled resonance modes and directional narrow beam emission by means of photonic nanojets [10]. These nanojets are the result of a microlensing effect. They emerge on the shadow side of the surface of a dielectric microcavity. The unique feature of nanojets is a directional beam with a beam waist smaller than the diffraction limit. The beam propagates over several wavelengths without significant beam divergence. Nanojets have been observed in optically coupled chains of microspheres [11]. We observed strongly directed far field emission in 3 -sphere, 5-sphere and 7-sphere structures, which we attribute to photonic nanojets. The directional emission from the PM matches the symmetry of the structure. The 7-sphere arrangement additionally shows an extraordinary hexagonal intensity distribution within the structure. We carried out spectral analysis of the scattered light from the multisphere structures. Independent of the photonic nanojets, we investigated if WGMs develop within the multisphere structures. We observed uncoupled modes as well as evidence of coherently coupled modes which are present in the PM.

Laser Resonators and Beam Control X, edited by Alexis V. Kudryashov, Alan H. Paxton, Vladimir S. Ilchenko, Proc. of SPIE Vol. 6872, 68720I, (2008) · 0277-786X/08/\$18 - doi: 10.1117/12.775751 


\section{EXPERIMENTAL METHOD}

We carried out spectral measurements by exciting the dye-coated spheres and taking the photoluminescence spectrum of a 3-sphere triangular PM and the 5- and 7-sphere cyclic PM. The 5-sphere cyclic PM is a ring structure formed by 5 microspheres while the 7-sphere PM is a ring structure with 6 outer spheres and one sphere in the centre. The experimental work carried out on the different PM configurations is discussed in the following sections. To form the symmetric PMs, the microspheres were placed on a solid Si-wafer substrate. Microspheres diluted in ethanol were dropcast on the substrate. Due to electrostatic force between the spheres, the microspheres conglomerate into different configurations. The microspheres used in this experiments are made of melamine formaldehyde latex $(\mathrm{n}=1.68)$ with a diameter of $5.374 \mu \mathrm{m} \pm 0.069 \mu \mathrm{m}$ according to specifications from MicroParticles $\mathrm{GmbH}$. The microspheres are coated with Rhodamine 6G fluorescence dye. The coated samples are stored in an aqueous or ethanol solution. The dye is used as a broadband emitter to probe the resonance spectrum of the PMs obtained by collecting the photoluminescence emission. The laser dye is optically pumped and the broad emission is coupled into the optical resonances of the spherical microcavity. The scattered emission from the PMs is collected to analyse the spectrum which represents the resonances. The microspheres on the substrate are in contact with each other when they form the PM structures. The CCD images of the emission field and the spectral measurements were taken with a commercial Renishaw microphotoluminescence/micro-Raman machine. In this setup, the laser light is focused perpendicular to the substrate from above the structure through a high-NA microscope objective $(100 \mathrm{x}, \mathrm{NA}=0.9)$. The scattered light is collected through the same objective operating in a confocal-like $180^{\circ}$ backscattering geometry and guided to the spectrometer and the CCD camera. The system collects all scattered light within the light cone of the objective in the focal plane. The substrate is placed on a computer controlled microstage, which allows an accurate spatial positioning of the laser focus in xyzdirection. The alignment is monitored with a CCD camera which shows the image of the optical microscope. When the photonic molecule sample on the substrate is selected, the focus position is aligned and the spectral scan is carried out. Different parameters such as the integration time, the spectral range and the pump laser intensity can be optimised in the control programme which operates the setup. A mechanical shutter avoids illumination of the sample between the measurements. The measured spectrum can then be further processed and saved as ASCII data.

\section{TRIANGULAR-SPHERE PHOTONIC MOLECULE}

The spectra of a single $5.374 \mu \mathrm{m}$ sphere and the coupled 3-sphere triangular structure are shown in Fig. 1. The spectrum in the upper graph of Fig. 1 reveals the resonance peaks of the TE and TM modes of a single microsphere with a nominal diameter of $5.374 \mu \mathrm{m}$. Detailed analysis of mode structure in these single microspheres is presented elsewere [12]. The sharpest and strongest resonances are TE modes. The fine unsplit peaks in the single sphere spectrum possess a linewidth of about $0.2 \mathrm{~nm}$. That corresponds to a Q-factor of about $Q \approx 2900$. It is noteworthy that the detected value of the Qfactor is strongly underestimated due to the limited resolution of the spectrometer $\left(>1 \mathrm{~cm}^{-1}\right)$. Another factor contributing to the line broadening is the contact with the substrate [13]. The dye coating on the sphere surface also reduces the Qfactor as the dye causes surface scattering and absorption [14]. The spectrum of the triangular structure is shown in the bottom graph of Fig. 1. The resonance mode distribution is rather complex, mainly due to higher order modes and the size difference of the microspheres, which means that the resonances of all three spheres are slightly shifted relative to each other. The spectrum reveals a series of additional fine peaks (double peaks marked with arrows in Fig. 1) partially grouped or overlapping each other with linewidths equal to single sphere resonances. The spectrum also shows resonances which can be assigned to modes in the single sphere spectrum. The additional resonances indicate coupled modes that have developed in the PM structure due to strong coherent coupling between the spherical microcavities. 

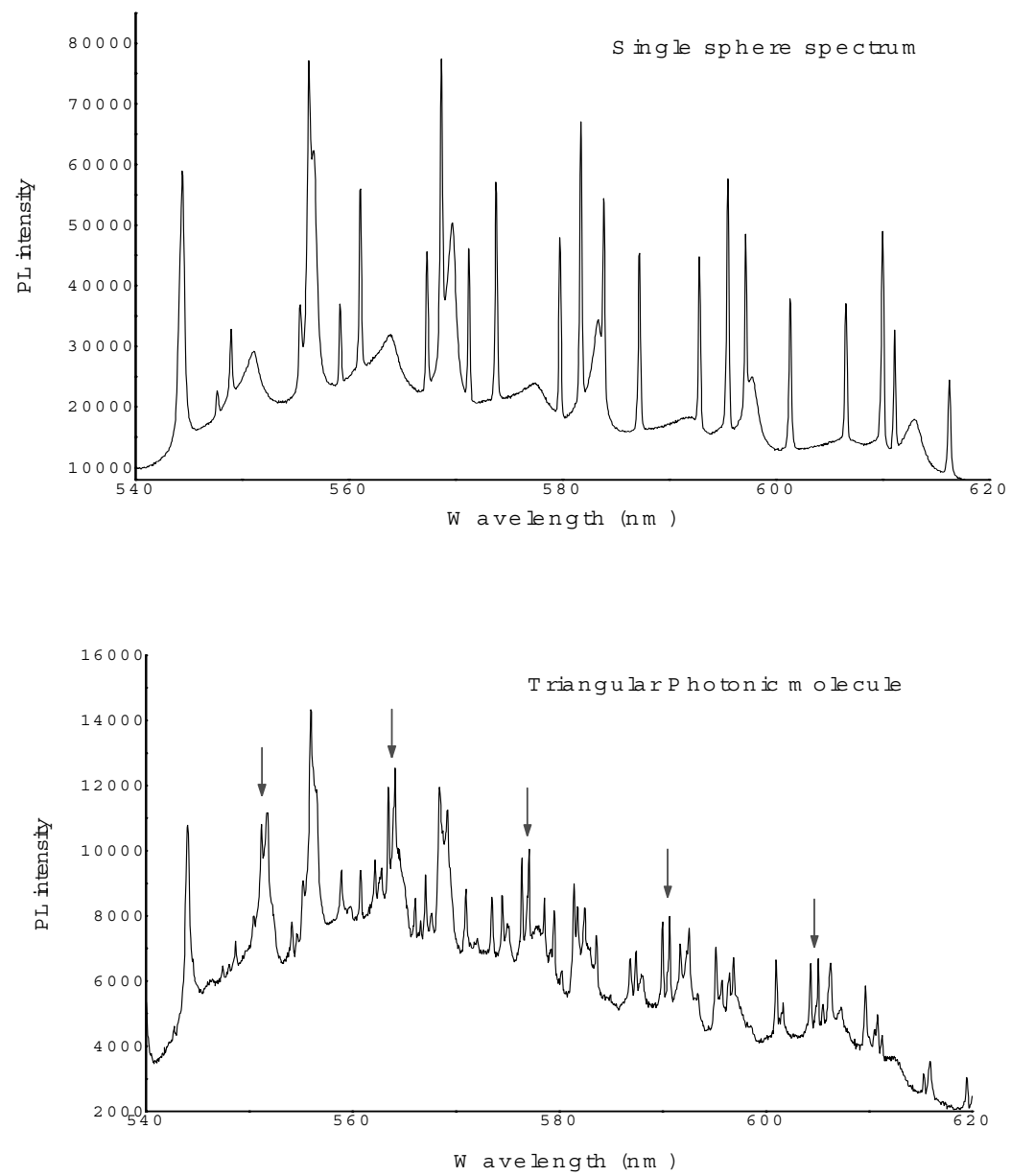

Fig. 1. Upper graph: Resonance spectrum of a single microsphere with a nominal diameter of $5.374 \mu m$ placed on a Si-wafer substrate. Bottom graph: Resonance spectrum of a triangular photonic molecule consisting of 3 microspheres. The arrows indicate possible coupled resonances.

The directional emission of a triangular PM is shown in Fig. 2. The laser is focused $7 \mu m$ above the Si-substrate in the zdirection centred in the crosshair as shown in the top inset of Fig. 1. The defocused beam allows coupling of the laser light into the whole structure, as the high-NA objective results in strong divergence of the focused beam. The position of the focus was adjusted for optimal coupling as monitored with the CCD camera. The bottom inset in the left image of Fig. 2 shows a merged image of the triangular structure in white light and the laser illuminated image. The overlapped image shows the position where the emission developed within the triangular PM is located. As the focus position of the laser and the focus plane of the CCD camera in the micro-photoluminescence setup cannot be adjusted separately, the image of the emission is not exactly in focus. Still, the image quality is sufficient to determine details in the scattered emission. The radii of the individual spheres are about $8 \lambda$. According to calculations based on Mie-theory [15], a sub- $\lambda$ nanojet on the surface of a microsphere can be obtained from spheres with a radius of about $\mathrm{R} \approx 5 \lambda$ and a refractive index of $n \approx 1.6$. Nanojets can also be formed with spheres of larger diameter $(R>20 \lambda)$ but with $n \approx 2$. With our microspheres, having a refractive index of $n=1.68$, the conditions for the formation of nanojets on the spheres surface are satisfied. The image in Fig. 2 clearly shows directional emission from all three microspheres at the outer surface of the individual spheres in the structure. Most remarkable are the emission spots with high intensity located near the surface of the spheres (see bottom inset). The reflections of the nanojets on the substrate clearly demonstrate the direction of the propagating beams. The inverted colour image in Fig. 2 on the right hand side also brings out features of the internal 
intensity distribution within the PM. The scattered light pattern within the PM resembles the typical distribution of the electromagnetic field for a nanojet in a microsphere which forms successive circular-shaped intensity minima and maxima. The observed intensity pattern shows similarities to field calculations carried out in $[15,16]$. It was shown in these publications that nanojets can be obtained with plane wave illumination of microspheres. Our experiments demonstrate that directional emission in the horizontal plane in three well defined directions with the triangular-sphere structure is possible under the condition of perpendicular illumination of the PM with a defocused laser beam. The photonic nanojets develop although it is not a vertical plane wave excitation technique [6].
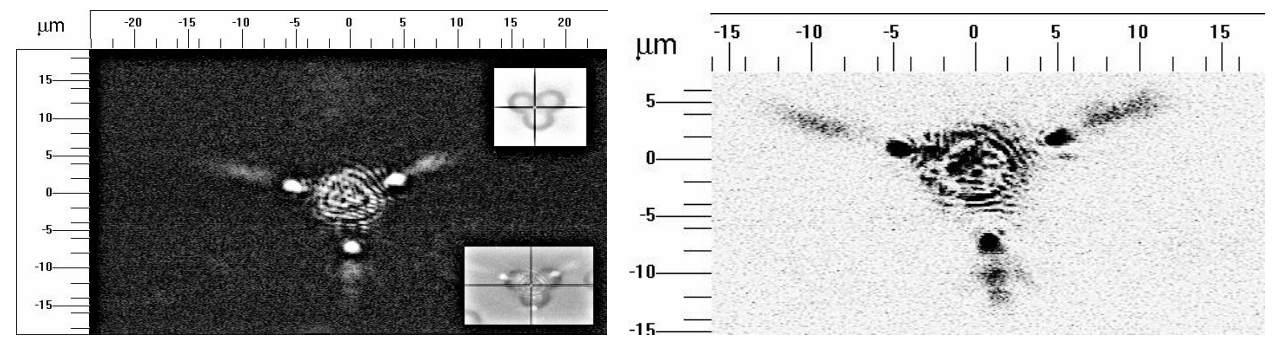

Figure 2. left: Photonic nanojets generated by laser illumination of a triangular photonic molecule. The far-field emission is visible due to reflection from the Si-substrate. The length scale in the frame is in micro-metres. Inset top right: Image of the tri-sphere photonic molecule in white light. The crosshair indicates the focus position of the laser. Inset bottom right: A merged image of the triangular structure under laser illumination and under white light, showing the spatial distribution of the emission within the structure. Right: Image shown in inverse colours. Dark colours indicate high intensities.

\section{5-SPHERE CYCLIC PHOTONIC MOLECULE}

The spectrum of the 5- sphere ring PM structure is shown in the bottom graph of Fig. 3. To illustrate the differences to the single sphere, the resonance spectrum of the single sphere is shown in Fig. 3 in the upper graph. In comparison with the 3-sphere triangular spectrum, we observe a rearrangement of the modes. Instead of narrow resonances, broad unevenshaped peaks develop. There are no sharp peaks which can be assigned to modes of the single sphere spectrum. Our experiment shows that the PM consisting of three symmetrically arranged microspheres can distribute a single excitation beam into three well-defined directional beams. To study the possibility of symmetric emission from nanojets in more directions, we formed structures consisting of a 5-sphere and a 7-sphere cyclic PM. As Fig. 4 shows, the 5-sphere ring also emits 5 nanojets at each sphere surface symmetric to the shape of the PM. In this structure, the laser is focused in the crosshair $6.5 \mu \mathrm{m}$ above the $\mathrm{Si}$ - substrate. The reflections of the nanojets on the substrate appear at around a distance of 3 to $4 \mu \mathrm{m}$ from the microspheres. Each sphere also shows the bright intensity spot at the surface from where the photonic nanojets emerge outwards symmetric to the structure. The directed emission suggests the possible use of these photonic structures as a beam splitter with a number of ports corresponding to the number of spheres arranged in the photonic molecule. Photonic nanojets may allow coupling of light from the PM into other photonic micro- or nanostructure such as coupled resonant optical waveguides or nanostructures such as nanocoaxial cables [17], which are sub-wavelength optical waveguides. This would be possible not only with sub-wavelength spatial accuracy, but also with extremely high intensities in comparison with conventional sub-wavelength coupling techniques using scanning-near-field optical microscopy (SNOM)-tips. 

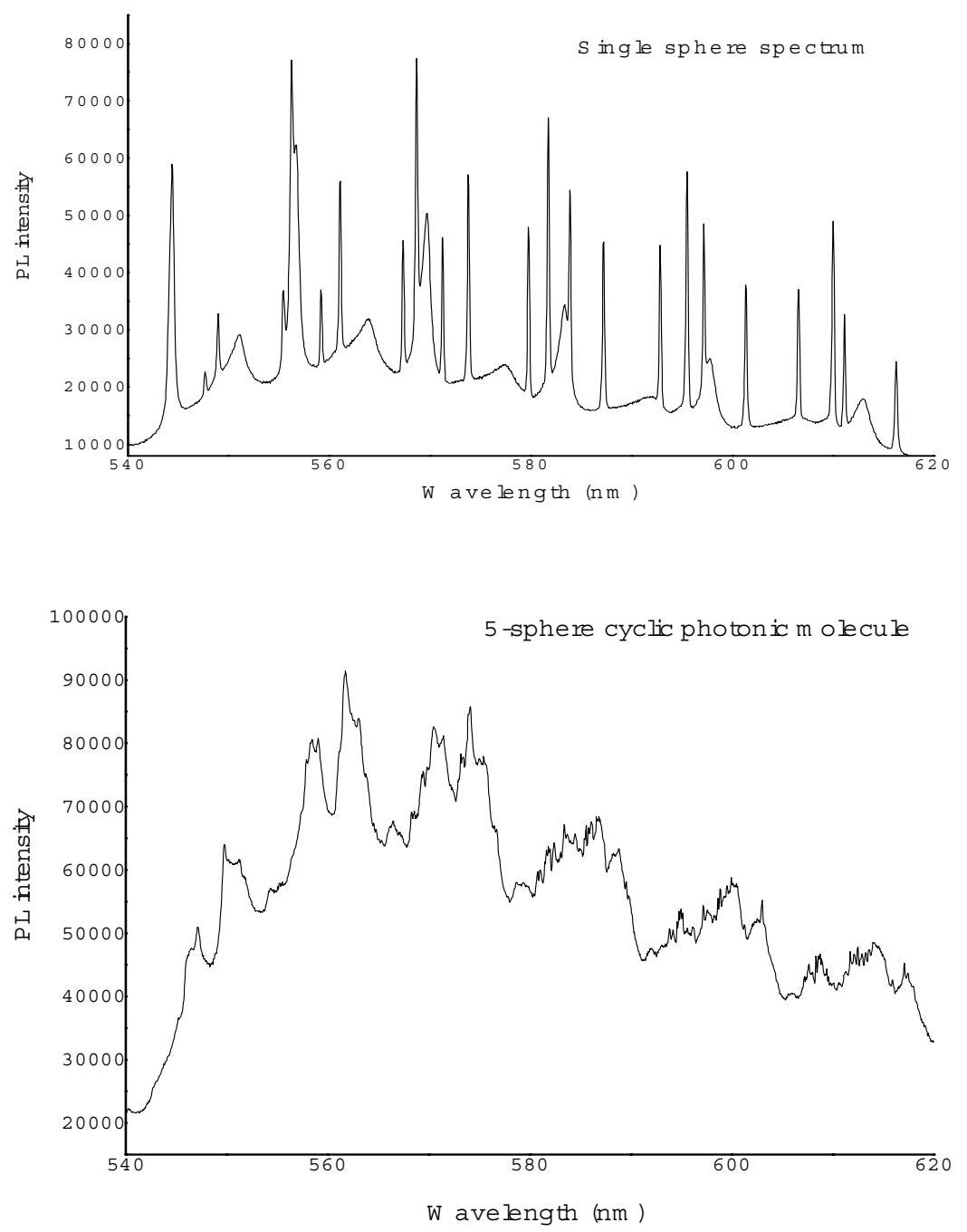

Fig.3. Upper graph: Resonance spectrum of a single microsphere with a nominal diameter of $5.374 \mu m$ placed on a Si-wafer substrate. Bottom graph: Resonance spectrum of a 5-sphere cyclic photonic molecule.

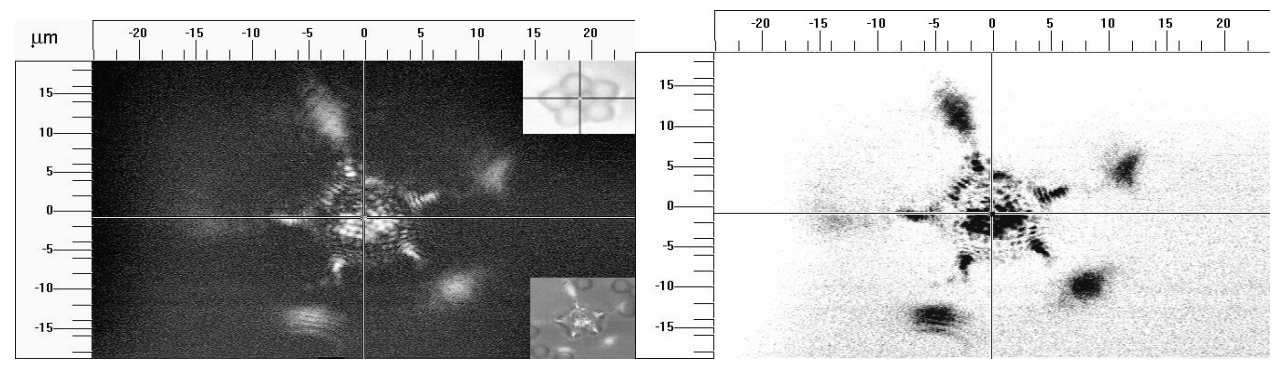

Fig. 4. left: 5-sphere ring photonic molecule without a sphere in the centre. The photonic molecule shows symmetrical directional emission. Inset top right: Image of 5-sphere cyclic photonic molecule in white light. The crosshair indicates the focus position of the laser. Inset bottom right: A merged image of the 5-sphere cyclic photonic molecule under laser illumination and under white light, showing the spatial distribution of the emission within the structure. Right: Image shown in inverse colours. Dark colours indicate high intensities. 


\section{7-SPHERE CYCLIC PHOTONIC MOLECULE}

The bottom graph in Fig. 5 shows the spectrum of the 7-sphere PM structure. The broad peaks are more pronounced and smoother in shape. The peaks possess a linewidth of about $2 \mathrm{~nm}$. That means that the Q-factor is about one magnitude smaller than in the single sphere modes. Due to the size disorder of all 7 spheres, the linewidth is altered by inhomogeneous broadening as a result of the overlapping resonances. The individual modes are also broadened as a result of the direct contact between the spheres. The more spheres are arranged in a PM, the more contact regions between the spheres occur and as a result, a lowering of the Q-factor of the optical resonances takes place. The most interesting feature of the 7-sphere system is that the modes are evenly spaced across the spectrum, indicating clearly that coherent mode coupling within the photonic molecule is indeed occurring. The most remarkable PM in this work was formed with 7 microspheres. Six of the spheres were arranged in a ring while one sphere was placed in the centre. The illuminated structure is shown in Fig 6. In agreement with the photonic molecules shown above, we observed nanojet emission from each of the outside spheres. The reflection of the nanojet beams on the Si-substrate shows a round and less elongated shape compared with the 5 microsphere PM. The most outstanding feature of this structure is the field intensity within the PM. The detected light forms a hexagonal shape with a strong resemblance to the electron cloud distribution in a chemical benzene molecule. Even the edges appear sharp in a clear hexagonal closed ring. The structure was illuminated in the centre with a defocused beam $8 \mu \mathrm{m}$ above the substrate. Spectral analysis of the scattered light should reveal if the illuminated PM develops strong optical coupling between the spheres. Studies of the spectral data of photonic benzene based on coupled defects in a photonic crystal were carried out by Lin [18] and Sievenpiper and coworkers [19]. The molecule orbitals are similar to the anti-bonding and bonding modes in coherently coupled microcavities. Calculations for photonic benzene [18 reveal a frequency spectrum similar to the $\pi$-molecule orbitals and the $\sigma$-molecule orbitals in the chemical molecule. The optical resonances show non-degenerate and degenerate modes in the spectrum of the coherently coupled resonators [19. Spectral analysis of our 7-sphere structure could clarify if the hexagonal intensity distribution is related to supermodes. This would require a mode mapping method [8] where spatial and spectral filtering allows studying of the spatial intensity distribution within the PM for particular modes. Our initial spectral analysis presented here, shows the spectrum of the scattered light from the whole PM. The not-spatially filtered spectrum provides a general evaluation of the optical resonances in the different PM structures with regards to uncoupled and coupled modes. We are currently developing a setup which allows the controlled separation of the microspheres within the PM as well as a method to reduce the dissipation of light into the substrate. The aim is to achieve the highest possible Q-factor of the individual modes. This would result in higher Q-factor of the coupled modes of the PM. Under optimised conditions, a strong enhancement of the Q-factor is expected when microcavities forming a PM are slightly separated from each other [3]. The Q-factor of specific coupled modes could increase up to 50 times compared with the uncoupled modes [3,5]. Applying sphere separation to our PMs could potentially bring out more pronounced characteristics of the resonance spectrum related to coherent coupling. 

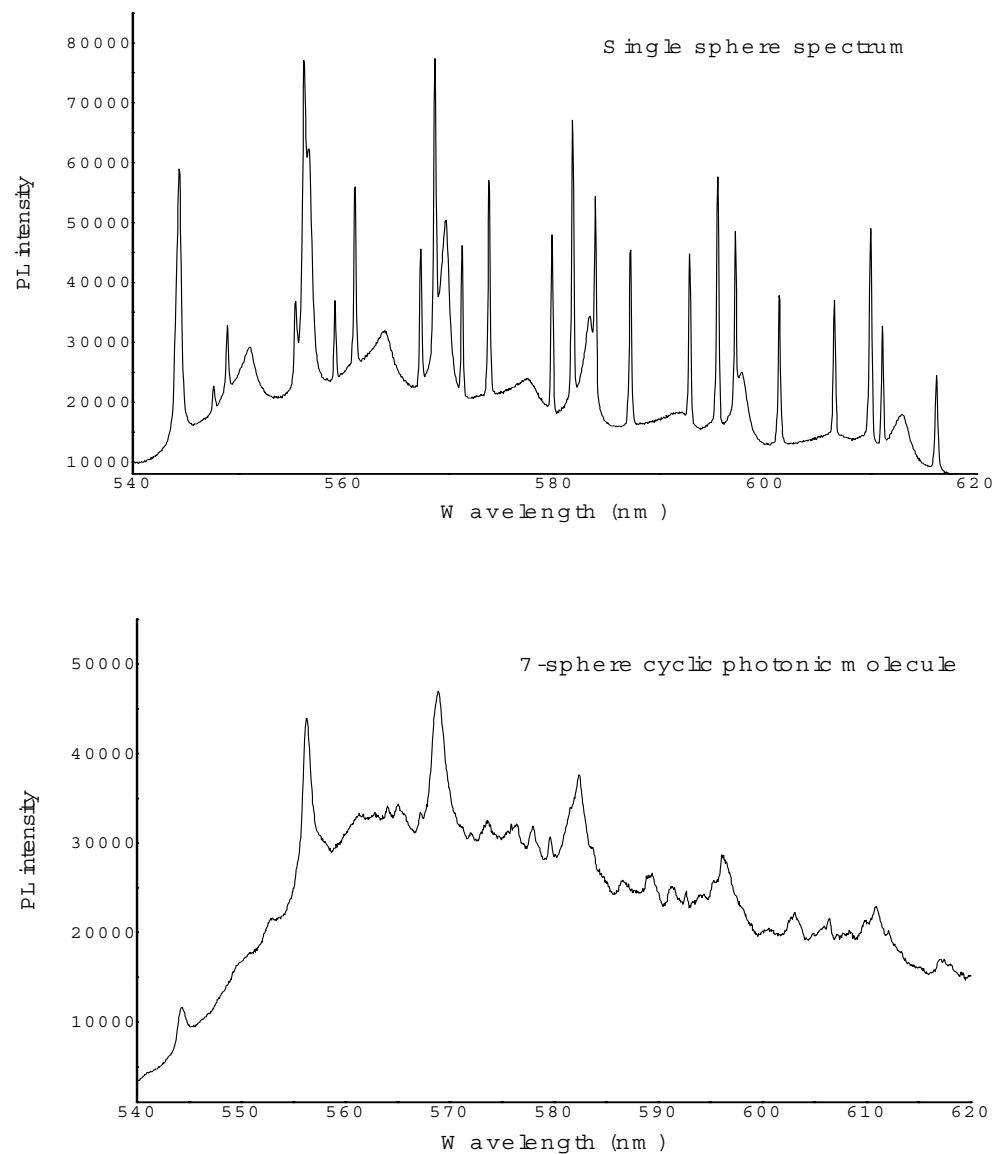

Fig. 5. Upper graph: Resonance spectrum of a single microsphere with a nominal diameter of $5.374 \mu m$ placed on a Si-wafer substrate. Bottom graph: Resonance spectrum of a 7-sphere cyclic photonic molecule structure with a centre sphere.

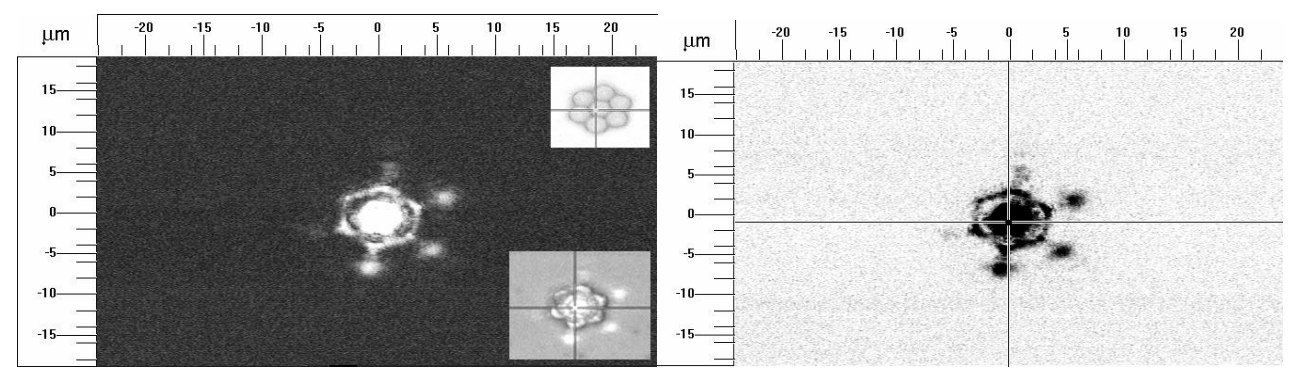

Fig. 6. left: A 7-sphere cyclic photonic molecule which shows strong similarities to a benzene ring structure of a chemical benzene molecule. Inset top right: Image of 7-sphere cyclic photonic molecule in white light. The crosshair indicates the focus position of the laser. Inset bottom right: A merged image of the 7-sphere cyclic photonic molecule under laser illumination and under white light, showing the spatial distribution of the emission within the structure. Right: Image shown in inverse colours. Dark colours indicate high intensities. 


\section{CONCLUSIONS}

In conclusion, the spectral analysis of the scattered light revealed WGMs in the spectrum attributed to overlapping uncoupled modes and the formation of broad peaks, indicating coherent coupling between the spheres of the PM. We have demonstrated directional emission from symmetric PMs of various shapes which we attribute to the formation of photonic nanojets. The PMs were illuminated perpendicular to the molecule plane with a defocused laser beam. Remarkable far-field emission was observed in these symmetric PM structures including a photonic benzene-like structure.

\section{ACKNOWLEDGMENTS}

This work has been supported by Science Foundation Ireland through the CRANN Research Centre, project IN07 "Cyclic Photonic Molecules".

\section{REFERENCES}

$1 \quad$ K. J. Vahala, "Optical microcavities," Nature 424, 839 (2003).

2 M. Bayer, T. Gudbrod, J. P. Reithmaier, A. Forchel, T. L. Reinecke, and P. A. Knipp, "Optical modes in photonic molecules," Phys. Rev. Lett. 81, 2582 (1998).

3 S. V. Boriskina, "Theoretical prediction of a dramatic Q-factor enhancement and degeneracy removal of whispering gallery modes in symmetrical photonic molecules," Opt. Lett. 31, 338 (2006).

E. I. Smotrova, A. I. Nosich, T. M. Benson, and P. Sewell, "Threshold reduction in a cyclic photonic molecule laser composed of identical microdisks with whispering-gallery modes", Opt. Lett. 31, 921 (2006).

5 S. V. Boriskina, "Spectrally engineered photonic molecules as optical sensors with enhanced sensitivity: a proposal and numerical analysis," J. Opt. Soc. Am. B 23, 1565 (2006).

6 S. V. Boriskina, T. M. Benson, and P. Sewell, "Photonic molecules made of matched and mismatched microcavities: new functionalities of microlasers and optoelectronic components," Proceedings of SPIE, Laser Resonators and Beam Control IX 6452, (2007).

7 V. N. Astratov, J. P. Franchak, and S. P. Ashili, "Optical coupling and transport phenomena in chains of spherical dielectric microresonators with size disorder," Appl. Phys. Lett. 85, 5508 (2004).

8 B. Moeller, U. Woggon, and M. V. Artemyev, "Photons in coupled microsphere resonators," J. Opt. A: Pure Appl. Opt. 8, S113 (2006).

9 Y. P. Rakovich, J. F. Donegan, M. Gerlach, A. L. Bradley, T. M. Connolly, J. J. Boland, N. Gaponik, and A. Rogach, "Fine structure of coupled optical modes in photonic molecules," Phys. Rev. A 70, 051801 (2004).

10 Z. Chen, A. Taflove, and V. Backman, "Photonic nanojet enhancement of backscattering of light by nanoparticles: a potential novel visible-light ultramicroscopy technique," Opt. Exp. 12, 1214 (2004).

11 A. M. Kapitonov and V. N. Astratov, "Observation of nanjet-induced modes with small propagation losses in chains of coupled spherical cavities," Opt. Lett. 32, 409 (2007).

12 Y.P. Rakovich, L. Yang, E.M. McCabe, J.F. Donegan, T. Perova, A. Moore, N. Gaponik, and A. Rogach, "Whispering Gallery Mode Emission from a Composite System of CdTe Nanocrystals and a Spherical Microcavity," Sem. Sci. Tech. 18, 914 (2003).

13 N. Le Thomas, U. Woggon, W. Langbein, and M. V. Artemyev, "Effect of a dielectric substrate on whisperinggallery-mode sensors," J. Opt. Soc. Am. B 23, 2361 (2006).

14 P. Chylek, H.-B. Lin, J. D. Eversole, and A. J. Campillo, "Absorption effects on microdroplet resonant emission structure," Opt. Lett. 16, 1723 (1991).

15 S. Lecler, Y. Takakura, and P. Meyrueis, "Properties of a three-dimensional photonic jet," Opt. Lett. 30, 2641 (2005).

16 A. V. Itagi and W. A. Challenger, "Optics of photonic nanojets," Opt. Soc. Am. A 22, 2847 (2006).

17 T. Thio, "Coaxing light into small spaces," Nature Nanotechnology 2, 136 (2007).

18 B. S. Lin, "Variational analysis for photonic molecules: Application to photonic benzene waveguides," Phys. Rev. E 68, 0336611 (2003).

${ }_{19}$ D. F. Sievenpiper, M. E. Sickmiller, and E. Yablonovitch, “3D Wire Mesh Photonic Crystals," Phys. Rev. Lett. 76, 2480 (1996). 